also Selecta, Paris, 1939, pp. 203-233.

5. G. de Rham, Sur l'analysis situs des variêtés à $n$ dimensions. J. Math. Pures Appl. vol. 10 (1931) pp. 115-200.

6. H. Hopf und H. Samelson, Ein Satz über die Wirkungsräume geschlossener Liescher Gruppen, Comment Math. Helv. vol. 13 (1941), pp. 240-251.

7. E. Stiefel, Ueber eine Beziehung zwischen geschlossenen Lie'schen Gruppen und . . . Comment. Math. Helv. vol. 14 (1942), pp. 350-380.

8. E. Witt, Spiegelungsgruppen und Aufzählung halbeinfacher Lie'scher Ringe, Abh. Math. Sem. Hamburgischen Univ. vol. 14 (1941) pp. 289-322.

ZÜRICH, SwITZERLAND

\title{
A NOTE ON LEAST COMMON LEFT MULTIPLES
}

\section{B. M. STEWART}

1. Introduction. Consider $n$-by- $n$ matrices $A, B, \cdots$ with elements in a principal ideal ring and recall the following definitions. If $A=B C$, then $A$ is a left multiple of $C$ and $C$ is a right divisor of $A$. If $A=R D$ and $B=P D$, then $D$ is a common right divisor of $A$ and $B$; if, furthermore, $D$ is a left multiple of every common right divisor of $A$ and $B$, then $D$ is a greatest common right divisor of $A$ and $B$. If $M=P A=Q B$, then $M$ is a common left multiple of $A$ and $B$; if, furthermore, $M$ is a right divisor of every common left multiple of $A$ and $B$, then $M$ is a least common left multiple of $A$ and $B$. If $F E=I$, where $I$ is the identity matrix, then $E$ is a unimodular matrix. If $E$ is unimodular, then $E A$ is a left associate of $A$.

The basic tool in the following constructions is the theorem ${ }^{1}$ that any given matrix $A$ is the left associate of a uniquely determined matrix $A_{1}$, known as the Hermite canonical triangular form, having zeros above the main diagonal, having elements below the main diagonal in a prescribed residue class modulo the diagonal element above, having each diagonal element in a prescribed system of nonassociates, and if a diagonal element is zero, having the corresponding row all zero.

C. C. MacDuffee has presented the following method, ${ }^{2}$ due in essence to E. Cahen and A. Chatelet, for finding a greatest common

Presented to the Society, September 7, 1948; received by the editors April 30, 1948.

${ }^{1}$ C. C. MacDuffee, Matrices with elements in a principal ideal ring, Bull. Amer. Math. Soc. vol. 39 (1933) pp. 570-573.

${ }^{2}$ C. C. MacDuffee, loc. cit. p. 573. 
right divisor $D$ and a least common left multiple $M$ of two given matrices $A$ and $B$. Consider $2 n$-by- $2 n$ matrices written in the form of $n$-by- $n$ blocks. Use the theory of the Hermite form, as applied to the $2 n$-by- $2 n$ matrices, to write the following equations.

$$
\begin{gathered}
\left(\begin{array}{ll}
R & S \\
P & Q
\end{array}\right)\left(\begin{array}{ll}
A & 0 \\
B & 0
\end{array}\right)=\left(\begin{array}{ll}
D & 0 \\
0 & 0
\end{array}\right), \quad\left(\begin{array}{ll}
R^{\prime} & S^{\prime} \\
P^{\prime} & Q^{\prime}
\end{array}\right)\left(\begin{array}{ll}
R & S \\
P & Q
\end{array}\right)=\left(\begin{array}{ll}
I & 0 \\
0 & I
\end{array}\right), \\
\left(\begin{array}{ll}
A & 0 \\
B & 0
\end{array}\right)=\left(\begin{array}{ll}
R^{\prime} & S^{\prime} \\
P^{\prime} & Q^{\prime}
\end{array}\right)\left(\begin{array}{ll}
D & 0 \\
0 & 0
\end{array}\right) .
\end{gathered}
$$

Since first: $A=R^{\prime} D$ and $B=P^{\prime} D$, and second: $R A+S B=D$, it follows that $D$ is a greatest common right divisor of $A$ and $B$, with no restrictions on $A$ and $B$.

With the rather severe restriction that both $A$ and $B$ be nonsingular, it has been shown ${ }^{3}$ that the matrix $M=P A=-Q B$, obtained from (1), is a least common left multiple of $A$ and $B$.

It is the purpose of this note to show by a different proof that the stated method for finding $M$ is correct for a much larger set of matrices $A$ and $B$-namely, whenever $D$ is nonsingular, that is, whenever the row space of the union of $A$ and $B$ has the rank $n$, thus including, in addition to the case that both $A$ and $B$ are nonsingular, the case when either $A$ or $B$ is nonsingular and many other possibilities. Furthermore, in the exceptional case when $D$ is singular, there is presented a method for finding an $M$ that may be considered a natural modification of the method based on the equations (1). In conclusion it is noted that a theorem of E. Steinitz guarantees that both $D$ and $M$ are unique up to a unimodular left factor.

For completeness it should be noted that a method for finding the least common left multiple of two matrices when the matrices have elements in a field has been given by C. C. MacDuffee, ${ }^{4}$ but the method is not applicable to the case where the elements are limited to a principal ideal ring.

2. When $D$ is nonsingular. In terms of the notation explained in the introduction the following theorem may be stated:

Theorem 1. In the matric equation

$$
\left(\begin{array}{ll}
R & S \\
P & Q
\end{array}\right)\left(\begin{array}{ll}
A & 0 \\
B & 0
\end{array}\right)=\left(\begin{array}{ll}
D & 0 \\
0 & 0
\end{array}\right)
$$

${ }^{3}$ C. C. MacDuffee, loc. cit. p. 574.

${ }^{4}$ C. C. MacDuffee, Vectors and matrices, Carus Monograph, 1943, pp. 100-102. 
where the matrix involving $R, S, P, Q$ is unimodular, the matrix $D$ is an all cases a greatest common right divisor of $A$ and $B$; and if $D$ is nonsingular, then the matrix $M=P A=-Q B$ is a least common left multiple of $A$ and $B$.

That $D$ is in all cases a greatest common right divisor has been shown above. To show that $M$, which is obviously a common left multiple of $A$ and $B$, is a least common left multiple, let $M_{1}=U A$ $=-V B$ be any common left multiple of $A$ and $B$. Use the matrices determined in (1) to write the following equations:

$$
\begin{gathered}
\left(\begin{array}{ll}
R & S \\
U & V
\end{array}\right)\left(\begin{array}{ll}
A & 0 \\
B & 0
\end{array}\right)=\left(\begin{array}{ll}
D & 0 \\
0 & 0
\end{array}\right), \\
\left(\begin{array}{ll}
R & S \\
U & V
\end{array}\right)\left(\begin{array}{ll}
R^{\prime} & S^{\prime} \\
P^{\prime} & Q^{\prime}
\end{array}\right)\left(\begin{array}{ll}
D & 0 \\
0 & 0
\end{array}\right)=\left(\begin{array}{ll}
D & 0 \\
0 & 0
\end{array}\right) .
\end{gathered}
$$

Consider the most general solution of the equation

$$
\left(\begin{array}{cc}
X & W \\
Y & T
\end{array}\right)\left(\begin{array}{ll}
D & 0 \\
0 & 0
\end{array}\right)=\left(\begin{array}{ll}
D & 0 \\
0 & 0
\end{array}\right)
$$

Here $W$ and $T$ are arbitrary, but $X$ and $Y$ must be chosen so that

$$
X D=D, \quad Y D=0 .
$$

Subject to the conditions (3) the following equations must hold:

$$
\left(\begin{array}{ll}
R & S \\
U & V
\end{array}\right)\left(\begin{array}{ll}
R^{\prime} & S^{\prime} \\
P^{\prime} & Q^{\prime}
\end{array}\right)=\left(\begin{array}{cc}
X & W \\
Y & T
\end{array}\right), \quad\left(\begin{array}{ll}
R & S \\
U & V
\end{array}\right)=\left(\begin{array}{cc}
X & W \\
Y & T
\end{array}\right)\left(\begin{array}{ll}
R & S \\
P & Q
\end{array}\right) .
$$

In particular, it appears that $U$ must have the form

$$
U=Y R+T P \text {. }
$$

But under the hypothesis that $D$ is nonsingular the only solution of $Y D=0$ is $Y=0$. Hence it follows in this case that $U=T P$; then from $M_{1}=U A=T P A=T M$ it follows that $M=P A=-Q B$ is indeed a least common left multiple of $A$ and $B$ and the proof of the theorem is complete.

3. When $D$ is singular. If the matrix $D$ is singular it is easy to construct examples in which the matrix $P A$ of Theorem 1 is not a least common left multiple of $A$ and $B$. Hence the construction proposed in the following theorem has some interest.

Theorem 2. Let $A_{1}$ and $B_{1}$ be the Hermite forms of $A$ and $B$, respectively. In the matric equation 


$$
\left(\begin{array}{ll}
R^{*} & S^{*} \\
P^{*} & Q^{*}
\end{array}\right)\left(\begin{array}{ll}
A_{1} & 0 \\
B_{1} & 0
\end{array}\right)=\left(\begin{array}{ll}
D & 0 \\
0 & 0
\end{array}\right)
$$

where the matrix involving $R^{*}, S^{*}, P^{*}, Q^{*}$ is unimodular and the matrix involving $D$ is in Hermite form, let it be agreed, since this is a possible procedure, that no operations are performed upon the rows corresponding to the zero rows of $D$. Then the matrix $M^{*}=P^{*} A_{1}=-Q^{*} B_{1}$ is a least common left multiple of $A$ and $B$.

It follows readily that if $E_{1}$ and $E_{2}$ are unimodular, then a least common left multiple $M^{*}$ of $A_{1}=E_{1} A$ and $B_{1}=E_{2} B$ is also a least common left multiple of $A$ and $B$. First: from $M^{*}=K A_{1}=L B_{1}$ it follows that $M^{*}=K E_{1} A=L E_{2} B$; second: from $M_{1}=U A=V B$ it follows that $M_{1}=U F_{1} A_{1}=V F_{2} B_{1}$, where $F_{1} E_{1}=I=F_{2} E_{2}$, but since $M^{*}$ is a least common left multiple of $A_{1}$ and $B_{1}$, it follows that there exists a matrix $G$ such that $M_{1}=G M^{*}$. In particular $A_{1}$ and $B_{1}$ may be taken to be the Hermite forms of $A$ and $B$, respectively.

In the equation $\left(1^{\prime}\right)$ the ${ }^{*}$ notation has been adopted in describing the component blocks of the transforming matrix for two reasons: first: to indicate that the operations are to be made on the matrix involving $A_{1}$ and $B_{1}$ rather than $A$ and $B$; and second: to indicate that the operations are to be made in what may be described as one of the "simplest" ways. Certainly no zero row can appear in $D$ unless it is also a zero row of $A_{1}$ (the converse, of course, does not hold). Consequently, in obtaining the Hermite form involving $D$, it is quite unnecessary to operate on the rows $(z)$ which are zero rows of $D$, and this agreement results in a "simplest" transforming matrix. In fact the matrix $R^{*}$ must have each of its rows $(z)$ all zero except for a 1 in the diagonal position. Furthermore, the most general matrix $Y$ such that $Y D=0$ must have the form $Y=Z Y^{*}$, where $Z$ is arbitrary but $Y^{*}$ has a structure just like $R^{*}$ in its rows $(z)$ and zero rows elsewhere. Thus $Y^{*} R^{*}=Y^{*}$ and

$$
Y R^{*}=Z Y^{*} R^{*}=Z Y^{*}=Y .
$$

As observed above $A_{1}$ has zero rows in at least the rows (z), hence from $Y D=0$ it follows that $Y A_{1}=0$, and from (5) it follows that

$$
Y R^{*} A_{1}=Y A_{1}=0 .
$$

Let $M_{1}=U A_{1}=-V B_{1}$ be any common left multiple of $A_{1}$ and $B_{1}$. Starting from $\left(1^{\prime}\right)$ instead of (1), obtain the relations analogous to (2), (3), and (4). In particular, consider

$$
U=Y R^{*}+T P^{*} \text {. }
$$


From (6) it follows that $M_{1}=U A_{1}=\left(Y R^{*}+T P^{*}\right) A_{1}=T P^{*} A_{1}$. Hence with $M^{*}=P^{*} A_{1}=-Q^{*} B_{1}$ it follows that $M_{1}=T M^{*}$ and that $M^{*}$ is a least common left multiple of $A_{1}$ and $B_{1}$. Automatically $M^{*}$ is a least common left multiple of $A$ and $B$ and the proof of the theorem is complete.

It should be noted that Theorem 2 works in all cases and does not require $D$ to be singular. However the construction of Theorem 1 , when applicable, is much shorter, since the separate computation of $A_{1}$ and $B_{1}$ is avoided. A working procedure would be to start from $A$ and $B$ and compute $D$ in Hermite form; if $D$ has no zero rows, apply Theorem 1 ; if $D$ has zero rows (z), return to compute $A_{1}$ and $B_{1}$, find $D$ anew starting from $A_{1}$ and $B_{1}$ but not operating on the rows $(z)$, and then apply Theorem 2 .

\section{Uniqueness.}

Lemma. If $A=U B$ and $B=V A$, then there exists a unimodular matrix $E$ such that $B=E A$.

This lemma has been proved by E. Steinitz ${ }^{5}$ in a much more general setting - namely, for matrices whose elements are in a domain of integrity over the rational field, not necessarily a principal ideal ring. The proof is considerably simpler for the case of principal ideal rings. In fact by taking the proof for the case of matrices with elements in a field ${ }^{6}$ and merely replacing the word nonsingular by the word unimodular, a suitable proof for the case of principal ideal rings is easily made.

THEOREM 3. The greatest common right divisor $D$ and the least common left multiple $M$ of two given matrices $A$ and $B$ are uniquely determined up to unimodular left factors.

If $D$ and $D_{1}$ are two greatest common right divisors of $A$ and $B$, then each is a common left multiple of the other, say $D=U D_{1}$ and $D_{1}=V D$. Then by the lemma, $D$ and $D_{1}$ are left associates.

If $M$ and $M_{1}$ are two least common left multiples of $A$ and $B$, then each is a common right divisor of the other, say $M_{1}=U M$ and $M=V M_{1}$. Then by the lemma, $M$ and $M_{1}$ are left associates.

\section{Michigan State College}

\footnotetext{
${ }^{5}$ E. Steinitz, Math. Ann. vol. 71 (1911) pp. 328-354.
}

${ }^{6}$ C. C. MacDuffee, Vectors and matrices, pp. 45-46. 